\title{
Outage performance of relay-assisted transmissions in cognitive full-duplex relay networks
}

\author{
Hongbin Chen, Songgao Tan and Feng Zhao*
}

\begin{abstract}
Relay cooperation can enhance coverage, throughput, and reliability of wireless communication systems, thus attracting intense research interest in the past decade. However, most of them focused on half-duplex relay systems, which encounter loss of resource efficiency. With the progress of self-interference cancellation technologies, full-duplex relay systems come into sight. In this paper, outage performances of the primary system and the secondary system in a cognitive full-duplex relay network are analyzed, under the adverse effect of self-interference caused by the signals at the transmitting and receiving antennas of the relay. The full-duplex decode-and-forward relay assists the transmissions of the primary system and the secondary system where there is no direct link between the secondary transmitter and the secondary receiver. Simulation results show that better outage performance of the primary system is achieved compared with the cognitive relay network adopting half-duplex relaying. Moreover, communication of the secondary system is realized on the condition that the secondary transmission link is non-ideal.
\end{abstract}

Keywords: Full-duplex; Cognitive relay network; Self-interference; Outage probability

\section{Introduction}

Modern radio resource management is faced with the challenge of adapting to the increasing number of wireless applications and services on the limited amount of spectrum. However, as we know, most of the spectrum resources have been allocated under license [1]. The contradiction between the increasing demand and the limited spectrum resources inspires us to improve spectral efficiency. With this purpose, some new technologies have been proposed. For example, cooperative diversity was proposed to fight against channel fading and enhance throughput. The authors of [2,3] investigated the advantages of some cooperation schemes, and the authors of [4] studied an adaptive cooperation diversity scheme in multiple-relay cognitive radio networks. Cooperative relay forwards information from a source node to its destination node and emerges as an important technology to improve reliability, throughput, and coverage of wireless communication systems. Most of

\footnotetext{
* Correspondence: zhaofeng@guet.edu.cn

Key Laboratory of Cognitive Radio and Information Processing, Guilin

University of Electronic Technology, Ministry of Education, 1 Jinji Road, Guilin 541004, China
}

the studied relaying protocols are amplify-and-forward relaying and decode-and-forward relaying. In [5], the authors investigated achievable information rate and relay precoder design of multi-input multi-output amplifyand-forward relay networks under imperfect channel state information. The performance of decode-andforward relaying in spectrum sharing systems has also been widely studied [6].

Furthermore, interest in alternative models for sharing spectrum among wireless communication systems has increased [7]. Cognitive radios have been proposed to solve the spectrum resource scarcity problem. They were considered in the framework of hierarchical spectrum sharing where two different wireless communication systems are allowed to operate over the same part of spectrum even if they own different priorities. The system with higher priority is called primary system, and the system with lower priority is called secondary system. The secondary system transmits signals after detecting a spectrum hole or share the same frequency band with the primary system as long as the caused interference from the secondary system to the primary system is below a threshold. Cognitive relay network,

\section{包 Springer}


which combines cognitive radio and cooperative relaying, has attracted significant research interests $[8,9]$. It can improve not only the primary system performance but also the secondary system performance.

Basically, relay systems fall into two categories: halfduplex relaying and full-duplex relaying [10,11]. A large number of existing works on cooperative communications considered half-duplex relaying, where relays forward the source signal in orthogonal and dedicated channels [12]. The half-duplex relaying refers to the inability of the modems to transmit and receive signals in the same frequency band at the same time. This limitation results in inefficient use of system resources (bandwidth loss) [13]. In [14], analytic investigation on the outage performance of dual-hop multi-antenna amplifyand-forward relaying systems in the presence of interference was conducted. For both fixed-gain and variable-gain relaying schemes, exact analytic expressions of outage probability were derived. In our earlier work, a similar network model adopting half-duplex relaying was studied [15]. The authors proposed a two-phase protocol for relay-assisted transmissions in a cognitive relay network and calculated outage probabilities of the primary system and the secondary system separately.

As we all know, full-duplex transmission can obtain higher throughput than half-duplex transmission theoretically [16]. Full-duplex relaying refers to the ability of the relay to transmit and receive signals in the same frequency band and in the same time slot. This alternative efficiently uses the channel bandwidth, as it requires only one channel for end-to-end transmission [17]. So, full-duplex relaying has received much attention from both industry and academia recently. Pioneering works concentrated on the ideal scenario where the relay is able to transmit and receive signals simultaneously without any self-interference $[18,19]$. It is easy to learn that self-interference caused by the signal leakage between the relay output and input cannot be completely canceled in practice [20]. However, in the past, because of self-interference, full-duplex operation mode was considered impractical, but the improvement of antenna technology and signal processing techniques now make it feasible. Recently, several studies on full-duplex relaying take the effect of residual loop interference into account [21-27]. It should be pointed out that some of the above works only considered the non-cooperative dual-hop or multi-hop approach in full-duplex relaying with selfinterference [21,24] or completely ignored self-interference $[21,23,25]$. While these non-cooperative full-duplex relaying schemes allow the source to transmit continuously, their performances such as bit error rate and outage probability, have been shown to be severely degraded with the residual self-interference [23,24]. Recently, some authors have investigated the cooperative full-duplex relaying with the loop interference [26,27]. They studied the optimal power allocation scheme and the corresponding capacity limit of a full-duplex dual-hop amplify-and-forward relay system under residual self-interference in [26] and investigated the error and diversity performances of a full-duplex amplify-and-forward single relay system under the effect of residual self-interference in [27].

To date, there are few works on cognitive full-duplex relay systems. In [28], the cooperation between a primary system and a cognitive system in a cellular network was studied, where the cognitive base station relays the primary signal using an amplify-and-forward relaying or a decode-and-forward relaying protocol, and in turn, it can transmit its own signal. In [29], the authors dealt with the deployment of full-duplex relaying in amplifyand-forward cooperative networks with multi-antenna terminals and investigate a joint precoding/decoding design that maximizes the end-to-end performance. In [30], the authors proposed an optimal power allocation scheme for minimizing the overall outage probability in the cognitive full-duplex relay system, and then derived outage probabilities of the secondary user in the noiselimited and interference-limited environments. In this paper, we propose a spectrum-sharing protocol for a cognitive full-duplex relay network taking the selfinterference into account. The decode-and-forward relaying protocol is adopted since the relay has to decode the signals in order to perform self-interference cancellation. We consider the interference from the primary system to the secondary system, the interference from the secondary system to the primary system, and the self-interference between the relay output and the relay input. With full-duplex relaying, we try to decrease outage probability of the primary system versus the spectrum sharing protocol using half-duplex relaying. Meanwhile, the relay assists the transmission of the secondary system on the condition that there is no direct link between the secondary transmitter and the secondary receiver. Outage performances of the primary system and the secondary system are analyzed and simulated.

The rest of this paper is organized as follows. Section 2 describes the system model. The signal description in every transmission phase and outage probabilities of the primary and secondary systems are expressed in Section 3. Section 4 presents the simulation results and discussions, and Section 5 gives some concluding remarks.

\section{System model}

The cognitive full-duplex relay network model under consideration is shown in Figure 1. The primary system consists of a primary transmitter (PT) and a primary receiver (PR), while the secondary system consists of a secondary transmitter (ST) and a secondary receiver (SR). A full-duplex decode-and-forward relay (Relay) assists 


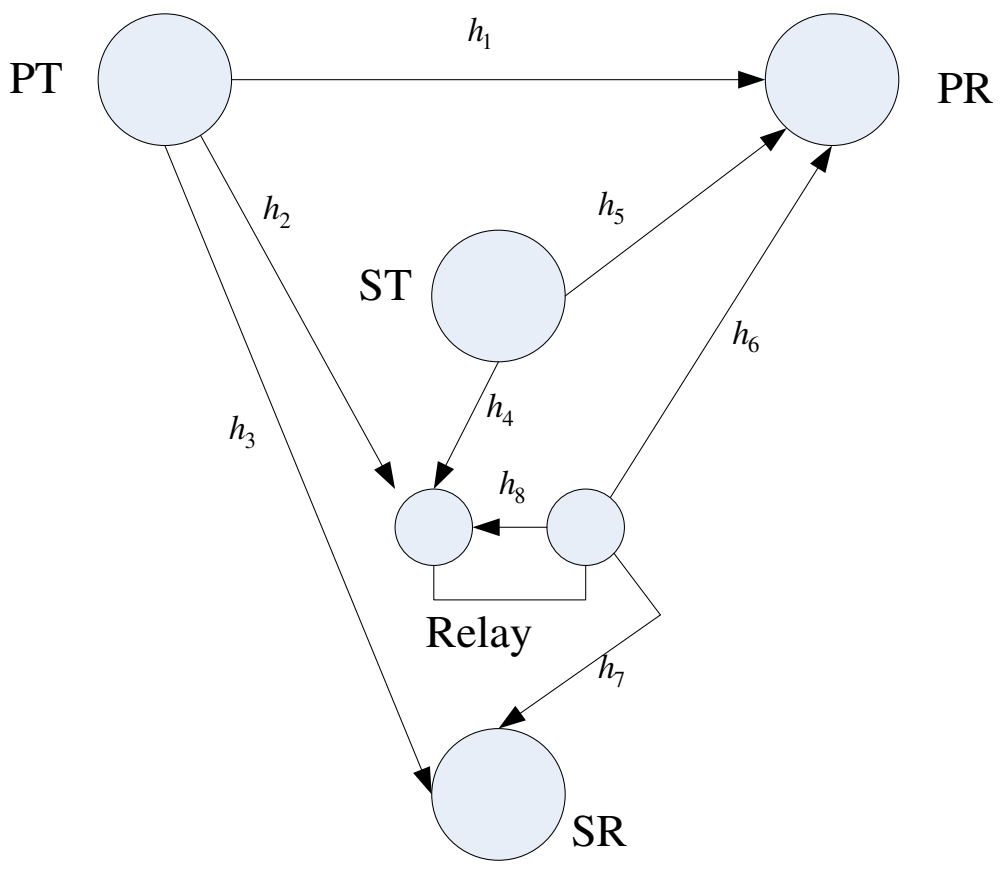

Figure 1 Cognitive full-duplex relay network model.

the transmissions of the primary system and the secondary system simultaneously. It is assumed that the secondary system follows the same radio protocols (e.g., coding, synchronization) as the primary system. In this cognitive relay network, outage performance of the primary system benefits from the assistance of the Relay, and communication of the secondary system is achieved with the assistance of the Relay when no direct link between the secondary transmitter and the secondary receiver is assumed [15].

Recall that in half-duplex relaying, the whole transmission process is divided into two phases. In the first transmission phase, the primary transmitter and the secondary transmitter transmit the primary signal and the secondary signal, respectively. The primary receiver, the Relay, and the secondary receiver receive the primary signal, while the Relay and the primary receiver receive the secondary signal. Then, the primary signal and the secondary signal are decoded and superimposed at the Relay. In the second transmission phase, the primary receiver and the secondary receiver receive a weighted linear composite signal which is transmitted by the Relay. At the primary receiver, the secondary signal is regarded as an interfering signal if the secondary signal is decoded successfully, and the primary signal is retrieved by maximal-ratio combining of the received signals in the two transmission phases. Similarly, the primary signal is regarded as an interfering signal at the secondary receiver if it is decoded successfully, and the secondary signal is retrieved from the signal which is sent from the
Relay. In full-duplex relaying, there is no transmission division and the Relay will transmit the weighted linear composite signal immediately after it receives the signals (the signal processing time is ignored). We should consider the self-interference caused by the signals at the transmitting and receiving antennas of the Relay. The Relay can decode the signals successfully provided that the amount of interference inflicted at the relay input is below a predetermined threshold, which is the maximum tolerable interference level at the relay input.

All the channels are assumed to experience Rayleigh block fading. The channel coefficients of the links PT $\rightarrow$ $\mathrm{PR}, \mathrm{PT} \rightarrow$ Relay, $\mathrm{PT} \rightarrow \mathrm{SR}, \mathrm{ST} \rightarrow$ Relay, $\mathrm{ST} \rightarrow \mathrm{PR}$, Relay $\rightarrow$ $\mathrm{PR}$, Relay $\rightarrow \mathrm{SR}$, and Relay output $\rightarrow$ Relay input are denoted by $h_{1}, h_{2}, h_{3}, h_{4}, h_{5}, h_{6}, h_{7}$, and $h_{8}$, respectively. Moreover, we have $h_{i} \sim C N\left(0, d_{i}^{-v}\right) \quad(i=1,2,3,4,5,6,7)$, and $h_{8} \sim C N\left(0, d_{8}^{-v 1}\right)$, where $v$ and $v 1$ are path loss exponents and $d_{i}$ is the normalized distance between the respective transmitter and receiver. This normalization is done with respect to the distance between the primary transmitter and the primary receiver, i.e., $d_{1}=1$. We also denote $r_{i}=\left|h_{i}\right|^{2}$. These channel gains are exponentially distributed random variables. Let $x_{p}$ and $x_{s}$ denote the primary signal and the secondary signal, respectively, with zero mean and $E\left\{x_{p}^{*} x_{p}\right\}=1, E\left\{x_{s}^{*} x_{s}\right\}=1$ where * denotes the conjugate operation. The transmission power at the primary transmitter, the secondary transmitter, and the Relay are denoted by $p_{p}, p_{s}$, and $p_{n}$ respectively. 
Notations: a circularly symmetric complex Gaussian random variable $z$ with variance $\sigma^{2}$ is denoted as $z \sim C N$ $\left(0, \sigma^{2}\right)$. An exponentially distributed random variable $x$ with mean $\frac{1}{\lambda}$ is denoted as $x \sim \varepsilon(\lambda) . E\{\cdot\}$ is used to denote the expectation operator.

\section{Signal description and outage performance analysis}

3.1 Outage performance of the cognitive full-duplex relay network (scheme A)

In this subsection, outage performances of the primary system and the secondary system in the cognitive fullduplex relay network will be analyzed. To better illustrate signal transmission and reception, the terms 'first transmission phase' and 'second transmission phase' are still used.

\subsubsection{Outage probability of the primary system}

In the first transmission phase, the primary transmitter transmits the primary signal $x_{p}$, while the primary receiver, the Relay, and the secondary receiver receive the signal, respectively. The received signals at the primary receiver, the Relay, and the secondary receiver are denoted by $y_{11}, y_{21}$, and $y_{31}$, respectively, which can be expressed as:

$$
y_{a 1}=\sqrt{p_{p}} h_{a} x_{p}+n_{a 1}
$$

where $a=1,2,3$. Here, $n_{a 1} \sim C N\left(0, \sigma^{2}\right)$ is an additive white Gaussian noise at the respective receiver in the first transmission phase. The achievable rate between the primary transmitter and the Relay is calculated by:

$$
R_{2}=\log _{2}\left(1+\frac{p_{p}\left|h_{2}\right|^{2}}{\sigma^{2}}\right)=\log _{2}\left(1+\frac{p_{p} r_{2}}{\sigma^{2}}\right)
$$

Likewise, the secondary transmitter transmits the secondary signal $x_{s}$, while the signal is received by the Relay as well as the primary receiver. The received signals at the Relay and the primary receiver are denoted by $y_{41}$ and $y_{51}$, respectively, which can be expressed as:

$$
y_{c 1}=\sqrt{p_{s}} h_{c} x_{s}+n_{c 1}
$$

where $c=4,5$. Here, $n_{c 1} \sim C N\left(0, \sigma^{2}\right)$ is an additive white Gaussian noise at the respective receiver. The achievable rate between the secondary transmitter and the Relay is denoted by $R_{4}$, and the one between the secondary transmitter and the primary receiver is denoted by $R_{5}$. They are calculated by:

$$
R_{d}=\log _{2}\left(1+\frac{p_{s} r_{d}}{\sigma^{2}}\right)
$$

where $d=4,5$.
Then, after reception in the first transmission phase, the Relay attempts to decode $x_{p}$ and $x_{s}$, respectively. If the Relay is able to decode both $x_{p}$ and $x_{s}$ successfully, a composite signal $x_{R}$ is generated by linearly combining the primary signal $x_{p}$ with power $\alpha p_{r}$ and the secondary signal $x_{s}$ with power $(1-\alpha) p_{n}$, where $\alpha(0 \leq \alpha \leq 1)$ is the power allocation factor at the Relay [8]. So, the composite signal is expressed as:

$$
x_{R}=\sqrt{\alpha p_{r}} x_{p}+\sqrt{(1-\alpha) p_{r}} x_{s}
$$

In the second transmission phase, $x_{R}$ is broadcasted and received by the primary receiver, the secondary receiver, and the relay input. The signal received by the relay input is expressed as:

$$
y_{32}=h_{8} x_{R}+n_{32}=\sqrt{\alpha p_{r}} h_{8} x_{p}+\sqrt{(1-\alpha) p_{r}} h_{8} x_{s}+n_{32}
$$

Here, $n_{32} \sim C N\left(0, \sigma^{2}\right)$ is an additive white Gaussian noise at the relay input. $y_{32}$ is regarded as the selfinterference at the relay input which affects the decoding at the Relay. The power of the self-interference can be calculated as $p$ loop $=\beta p_{r} r_{8}$, where $\beta(0 \leq \beta \leq 1)$ is the interference cancellation factor at the Relay. That is to say, if the self-interference is perfectly eliminated, $\beta=0$. But it is impossible in practical environments. If $p$ loop $\geq$ Ith, the Relay will fail to decode both $x_{p}$ and $x_{s}$, where Ith is the maximal interference that the Relay can tolerate. Likewise, the primary receiver receives the signal which is sent from the Relay if the Relay decodes both $x_{p}$ and $x_{s}$ successfully. Otherwise, the relay will keep silent. The received signal at the primary receiver is denoted by $y_{12}$, which can be expressed as:

$$
y_{12}=\sqrt{\alpha p_{r}} h_{6} x_{p}+\sqrt{(1-\alpha) p_{r}} h_{6} x_{s}+n_{12}
$$

Here, $n_{12} \sim C N\left(0, \sigma^{2}\right)$ is an additive white Gaussian noise at the primary receiver in the second transmission phase. Then, the interference component $\sqrt{(1-\alpha) p_{r}} x_{s}$ will be eliminated from $y_{12}$ on the condition that the primary receiver succeeds to decode $x_{s}$. So, we have $y_{12}^{*}=\sqrt{\alpha p_{r}} h_{6}$ $x_{p}+n_{12}$. Then, maximal-ratio combining will be applied to combine $y_{11}$ and $y_{12}^{*}$. The achievable rate, according to [12], is calculated by:

$$
R_{11}^{\mathrm{MRC}}=\log _{2}\left(1+\frac{p_{p} r_{1}}{\sigma^{2}}+\frac{\alpha p_{r} r_{6}}{\sigma^{2}}\right)
$$

If the primary receiver fails to decode $x_{s}$, the secondary signal will be regarded as noise at the primary receiver. 
Then, the signals $y_{11}$ and $y_{12}$ are also combined at the primary receiver by using maximal-ratio combining for the decoding of $x_{p}$, and the achievable rate is given by:

$$
R_{12}^{\mathrm{MRC}}=\log _{2}\left(1+\frac{p_{p} r_{1}}{\sigma^{2}}+\frac{\alpha p_{r} r_{6}}{(1-\alpha) p_{r} r_{6}+\sigma^{2}}\right)
$$

For the primary system, there are two cases where the primary signal is decoded successfully. The former is that $x_{p}$ is transmitted through the direct link from the primary transmitter to the primary receiver on the condition that the Relay keeps silent. In this case, the achieve rate between the primary transmitter and the primary receiver is given by $R_{1}=\log _{2}\left(1+\frac{p_{p} r_{1}}{\sigma^{2}}\right)$. The latter is that $x_{p}$ is transmitted from the relay-assisted link if the Relay decodes both $x_{p}$ and $x_{s}$ successfully. The former case includes four sub-cases, which are listed as follows: 1) If $p$ loop $\geq I$ th, the Relay fails to decode the signals, and $x_{p}$ is transmitted through the direct link; 2) $x_{p}$ is transmitted through the direct link on the condition that the Relay succeeds to decode $x_{p}$ and fails to decode $x_{s}$; 3) $x_{p}$ is transmitted through the direct link on the condition that the Relay fails to decode $x_{p}$ but succeeds to decode $x_{s}$;4) $x_{p}$ is transmitted through the direct link on the condition that the Relay fails to decode both $x_{p}$ and $x_{s}$. The latter case also includes two subcases: 1$)$ the Relay succeeds to decode both $x_{p}$ and $x_{s}$, and the primary receiver also decodes $x_{s}$ successfully. Then, the primary signal $x_{p}$ is transmitted through the relay-assisted link; 2) the Relay succeeds to decode both $x_{p}$ and $x_{s}$, but the primary receiver fails to decode $x_{s}$. Then, the primary signal $x_{p}$ is transmitted through the relay-assisted link.

The transmissions will be interrupted when the achievable rate is lower than the target rate. So, outage probability of the primary system with target rates $R_{p t}$ and $R_{s t}$ for the primary system and the secondary system, respectively, is calculated by:
According to [12], since $r_{1} \sim \varepsilon(1)$ and $r_{e} \sim \mathcal{E}\left(d_{e}^{\nu}\right) \quad(e=2,4$, $5,6,8)$, we have:

$$
P\left\{R_{1}>R_{p t}\right\}=P\left\{r_{1}>\frac{\rho_{1} \sigma^{2}}{P_{p}}\right\}=\exp \left(-\frac{\rho_{1} \sigma^{2}}{P_{p}}\right)
$$

$$
P\left\{R_{2}>R_{p t}\right\}=P\left\{r_{2}>\frac{\rho_{1} \sigma^{2}}{P_{p}}\right\}=\exp \left(-d_{2}^{\nu} \frac{\rho_{1} \sigma^{2}}{p_{p}}\right)
$$

$$
P\left\{R_{4}>R_{s t}\right\}=P\left\{r_{4}>\frac{\rho_{2} \sigma^{2}}{P_{s}}\right\}=\exp \left(-d_{4}^{v} \frac{\rho_{2} \sigma^{2}}{P_{s}}\right)
$$

$$
P\left\{R_{5}>R_{s t}\right\}=P\left\{r_{5}>\frac{\rho_{2} \sigma^{2}}{P_{s}}\right\}=\exp \left(-d_{5}^{v} \frac{\rho_{2} \sigma^{2}}{P_{s}}\right)
$$

$$
P\left\{p_{\text {loop }}>I \text { th }\right\}=P\left\{r_{8}>\frac{I \text { th }}{\beta p_{r}}\right\}=\exp \left(-d_{8}^{v} \frac{I \text { th }}{\beta p_{r}}\right)
$$

$$
\begin{aligned}
P\left\{R_{11}^{\mathrm{MRC}}>R_{p t}\right\}= & P\left\{\log _{2}\left(1+\frac{p_{p} r_{1}}{\sigma^{2}}+\frac{\alpha p_{r} r_{6}}{\sigma^{2}}\right)>R_{p t}\right\} \\
= & \mathrm{P}\left\{\left(p_{p} r_{1}+\alpha p_{r} r_{6}\right)>\rho_{1} \sigma^{2}\right\} \\
= & \exp \left(-\frac{\rho_{1} \sigma^{2}}{p_{p}}\right)+\left(-1+d_{6}^{\nu} \frac{p_{p}}{\alpha p_{r}}\right)^{-1} \\
& \times\left[\exp \left(-\frac{\rho_{1} \sigma^{2}}{p_{p}}\right)-\exp \left(-d_{6}^{\nu} \frac{\rho_{1} \sigma^{2}}{\alpha p_{r}}\right)\right]
\end{aligned}
$$

where $\rho_{1}=2^{R p t}-1, \rho_{2}=2^{R_{s t}}-1$. Assuming $p_{r}>>\sigma^{2}$, we have:

$$
P_{\text {out }}^{p}=1-\left[\begin{array}{l}
P\left\{p_{\text {loop }} \geq I \text { th }\right\} P\left\{R_{1}>R_{p t}\right\} \\
+P\left\{p_{\text {loop }}<I \text { th }\right\} \\
+P\left\{R_{2}>R_{p t}\right\} P\left\{R_{4}<R_{s t}\right\} P\left\{R_{1}>R_{p t}\right\} \\
+P\left\{p_{\text {loop }}<I \text { th }\right\} \\
P\left\{R_{2}<R_{p t}\right\} P\left\{R_{4}>R_{s t}\right\} P\left\{R_{1}>R_{p t}\right\} \\
+P\left\{p_{\text {loop }}<I \text { th }\right\} \\
P\left\{R_{2}<R_{p t}\right\} P\left\{R_{4}<R_{s t}\right\} P\left\{R_{1}>R_{p t}\right\} \\
+P\left\{p_{\text {loop }}<I \text { th }\right\} \\
P\left\{R_{2}>R_{p t}\right\} P\left\{R_{4}>R_{s t}\right\} P\left\{R_{5}>R_{s t}\right\} P\left\{R_{11}^{\mathrm{MRC}}>R_{p t}\right\} \\
+P\left\{p_{\text {loop }}<I \text { th }\right\} P\left\{R_{2}>R_{p t}\right\} P\left\{R_{4}>R_{s t}\right\} P\left\{R_{5}<R_{s t}\right\} P\left\{R_{12}^{\mathrm{MRC}}>R_{p t}\right\}
\end{array}\right]
$$




$$
\begin{aligned}
& P\left\{R_{12}^{\mathrm{MRC}}>R_{p t}\right\} \approx P\left\{\log _{2}\left(1+\frac{p_{p} r_{1}}{\sigma^{2}}+\frac{\alpha}{1-\alpha}\right)>R_{p t}\right\} \\
& =P\left\{r_{1}>\frac{\sigma^{2}}{p_{p}}\left(\rho_{1}-\frac{\alpha}{1-\alpha}\right)\right\} \\
& = \begin{cases}\exp \left[-\frac{\sigma^{2}}{p_{p}}\left(\rho_{1}-\frac{\alpha}{1-\alpha}\right)\right] & \leq \alpha \leq \hat{} \\
1 & \alpha \hat{\alpha} \leq \alpha \leq 1\end{cases} \\
& P_{\text {out }}^{p}=\left\{\begin{array}{cc}
P_{\text {out }}^{p, 1} & 0 \leq \alpha \leq \alpha^{\wedge} \\
P_{\text {out }}^{p, 2} & \alpha^{\wedge} \leq \alpha \leq 1
\end{array}\right. \\
& P_{\text {out }}^{p, 1}=1-\left\{\begin{array}{l}
\exp \left(-d_{8}^{v} \frac{I_{\text {th }}}{\beta p_{r}}\right) \exp \left(-\frac{\rho_{1} \sigma^{2}}{p_{p}}\right) \\
+\left[1-\exp \left(-d_{8}^{v} \frac{I_{\text {th }}}{\beta p_{r}}\right)\right] \exp \left(-d_{2}^{v} \frac{\rho_{1} \sigma^{2}}{p_{p}}\right)\left[1-\exp \left(-d_{4}^{v} \frac{\rho_{2} \sigma^{2}}{p_{s}}\right)\right] \exp \left(-\frac{\rho_{1} \sigma^{2}}{p_{p}}\right) \\
+\left[1-\exp \left(-d_{8}^{v} \frac{I_{\text {th }}}{\beta p_{r}}\right)\right]\left[1-\exp \left(-d_{2}^{v} \frac{\rho_{1} \sigma^{2}}{p_{p}}\right)\right] \exp \left(-d_{4}^{v} \frac{\rho_{2} \sigma^{2}}{p_{s}}\right) \exp \left(-\frac{\rho_{1} \sigma^{2}}{p_{p}}\right) \\
+\left[1-\exp \left(-d_{8}^{v} \frac{I_{\text {th }}}{\beta p_{r}}\right)\right]\left[1-\exp \left(-d_{2}^{v} \frac{\rho_{1} \sigma^{2}}{p_{p}}\right)\right]\left[1-\exp \left(-d_{4}^{v} \frac{\rho_{2} \sigma^{2}}{p_{s}}\right)\right] \exp \left(-\frac{\rho_{1} \sigma^{2}}{p_{p}}\right) \\
+\left[1-\exp \left(-d_{8}^{v} \frac{I_{\text {th }}}{\beta p_{r}}\right)\right] \exp \left(-d_{2}^{v} \frac{\rho_{1} \sigma^{2}}{p_{p}}\right) \exp \left(-d_{4}^{v} \frac{\rho_{2} \sigma^{2}}{p_{s}}\right) \exp \left(-d_{5}^{v} \frac{\rho_{2} \sigma^{2}}{p_{s}}\right) \\
{\left[\exp \left(-\frac{\rho_{1} \sigma^{2}}{p_{p}}\right)+\left(-1+d_{6}^{v} \frac{p_{p}}{\alpha p_{r}}\right)^{-1}\left(\exp \left(-\frac{\rho_{1} \sigma^{2}}{p_{p}}\right)-\exp \left(-d_{6}^{v} \frac{\rho_{1} \sigma^{2}}{\alpha p_{r}}\right)\right)\right]} \\
+\left[1-\exp \left(-d_{8}^{v} \frac{I_{\text {th }}}{\beta p_{r}}\right)\right] \exp \left(-d_{2}^{\nu} \frac{\rho_{1} \sigma^{2}}{p_{p}}\right) \exp \left(-d_{4}^{v} \frac{\rho_{2} \sigma^{2}}{p_{s}}\right) \\
{\left[1-\exp \left(-d_{5}^{v} \frac{\rho_{2} \sigma^{2}}{p_{s}}\right)\right] \exp \left[-\frac{\sigma^{2}}{p_{p}}\left(\rho_{1}-\frac{\alpha}{1-\alpha}\right)\right]}
\end{array}\right\}
\end{aligned}
$$

and:

$$
P_{\text {out }}^{p, 2}=1-\left\{\begin{array}{l}
\exp \left(-d_{8}^{v} \frac{I_{\text {th }}}{\beta p_{r}}\right) \exp \left(-\frac{\rho_{1} \sigma^{2}}{p_{p}}\right) \\
+\left[1-\exp \left(-d_{8}^{v} \frac{I_{\text {th }}}{\beta p_{r}}\right)\right] \exp \left(-d_{2}^{v} \frac{\rho_{1} \sigma^{2}}{p_{p}}\right)\left[1-\exp \left(-d_{4}^{v} \frac{\rho_{2} \sigma^{2}}{p_{s}}\right)\right] \exp \left(-\frac{\rho_{1} \sigma^{2}}{p_{p}}\right) \\
+\left[1-\exp \left(-d_{8}^{v} \frac{I_{\text {th }}}{\beta p_{r}}\right)\right]\left[1-\exp \left(-d_{2}^{v} \frac{\rho_{1} \sigma^{2}}{p_{p}}\right)\right] \exp \left(-d_{4}^{v} \frac{\rho_{2} \sigma^{2}}{p_{s}}\right) \exp \left(-\frac{\rho_{1} \sigma^{2}}{p_{p}}\right) \\
+\left[1-\exp \left(-d_{8}^{v} \frac{I_{\text {th }}}{\beta p_{r}}\right)\right]\left[1-\exp \left(-d_{2}^{v} \frac{\rho_{1} \sigma^{2}}{p_{p}}\right)\right]\left[1-\exp \left(-d_{4}^{v} \frac{\rho_{2} \sigma^{2}}{p_{s}}\right)\right] \exp \left(-\frac{\rho_{1} \sigma^{2}}{p_{p}}\right) \\
+\left[1-\exp \left(-d_{8}^{v} \frac{I_{\text {th }}}{\beta p_{r}}\right)\right] \exp \left(-d_{2}^{v} \frac{\rho_{1} \sigma^{2}}{p_{p}}\right) \exp \left(-d_{4}^{v} \frac{\rho_{2} \sigma^{2}}{p_{s}}\right) \exp \left(-d_{5}^{v} \frac{\rho_{2} \sigma^{2}}{p_{s}}\right) \\
{\left[\exp \left(-\frac{\rho_{1} \sigma^{2}}{p_{p}}\right)+\left(-1+d_{6}^{v} \frac{p_{p}}{\alpha p_{r}}\right)^{-1}\left(\exp \left(-\frac{\rho_{1} \sigma^{2}}{p_{p}}\right)-\exp \left(-d_{6}^{v} \frac{\rho_{1} \sigma^{2}}{\alpha p_{r}}\right)\right)\right]} \\
+\left[1-\exp \left(-d_{8}^{v} \frac{I_{\text {th }}}{\beta p_{r}}\right)\right] \exp \left(-d_{2}^{v} \frac{\rho_{1} \sigma^{2}}{p_{p}}\right) \exp \left(-d_{4}^{v} \frac{\rho_{2} \sigma^{2}}{p_{s}}\right) \\
{\left[1-\exp \left(-d_{5}^{v} \frac{\rho_{2} \sigma^{2}}{p_{s}}\right)\right]}
\end{array}\right\}
$$




\subsubsection{Outage probability of the secondary system}

In the first transmission phase, the secondary receiver receives the signal which is sent from the primary transmitter, and the received signal is expressed as $y_{31}=\sqrt{p_{p}}$ $h_{3} x_{p}+n_{31}$. The achievable rate between the primary transmitter and the secondary receiver is $R_{3}=\log _{2}$ $\left(1+\frac{p_{p} r_{3}}{\sigma^{2}}\right)$. In the second transmission phase, the secondary receiver receives the signal $x_{R}$ which is sent from the Relay on the condition that the Relay succeeds to decode both $x_{p}$ and $x_{s}$. The signal received at the secondary receiver is expressed as:

$$
\begin{aligned}
y_{22} & =h_{7} x_{R}+n_{22} \\
& =\sqrt{\alpha p_{r}} h_{7} x_{p}+\sqrt{(1-\alpha) p_{r}} h_{7} x_{s}+n_{22}
\end{aligned}
$$

Here, $n_{22} \sim C N\left(0, \sigma^{2}\right)$ is an additive white Gaussian noise at the secondary receiver in the second transmission phase. Assuming that the decoding of $x_{p}$ at the secondary receiver in the first transmission phase is successful, the interference component $\sqrt{\alpha p_{r}} h_{7} x_{p}$ will be removed from $y_{22}$ as an interfering signal. So we have $y_{22}^{*}=\sqrt{(1-\alpha) p_{r}} h_{7}$ $x_{s}+n_{22}$. The achievable rate between the Relay and the secondary receiver, conditioned on successful decoding of $x_{p}$ at both the Relay and the secondary receiver in the first transmission phase, is given by:

$$
R_{7}=\log _{2}\left(1+\frac{(1-\alpha) p_{r} r_{7}}{\sigma^{2}}\right)
$$

For the secondary system, an outage is declared except the only case where the Relay decodes both $x_{p}$ and $x_{s}$ successfully, and the secondary receiver also decodes $x_{p}$ successfully in the first transmission phase. So outage probability of the secondary system with the target rates $R_{p t}$ and $R_{s t}$ is calculated by:

$$
\begin{aligned}
& P_{\text {out }}^{s}= 1-P\left\{p_{\text {loop }}<I_{\text {th }}\right\} P\left\{R_{2}>R_{p t}\right\} P\left\{R_{3}>R_{p t}\right\} \\
& \times P\left\{R_{4}>R_{s t}\right\} P\left\{R_{7}>R_{s t}\right\} \\
&= 1-\left[1-\exp \left(-d_{8}^{v} \frac{I_{\text {th }}}{\beta p_{r}}\right)\right] \exp \left(-d_{2}^{v} \frac{\rho_{1} \sigma^{2}}{p_{p}}\right) \\
& \exp \left(-d_{3}^{v} \frac{\rho_{1} \sigma^{2}}{p_{p}}\right) \exp \left(-d_{4}^{v} \frac{\rho_{2} \sigma^{2}}{p_{s}}\right) \exp \left(-d_{7}^{v} \frac{\rho_{2} \sigma^{2}}{(1-\alpha) p_{r}}\right)
\end{aligned}
$$

\subsection{Outage performance of the cognitive half-duplex relay network (scheme B)}

In this subsection, outage performances of the primary system and the secondary system in the cognitive halfduplex relay network will be analyzed, and will be compared with those of the cognitive full-duplex relay network through simulations. Self-interference does not exist in cognitive half-duplex relay networks. So, outage probability of the primary system is given by:

$$
P_{\text {out }}^{p^{\prime}}=1-\left\{\begin{array}{c}
P\left\{R_{2}^{\prime}>R_{p t}\right\} P\left\{R_{4}^{\prime}<R_{s t}\right\} P\left\{\frac{1}{2} R_{1}>R_{p t}\right\} \\
+P\left\{R_{2}^{\prime}<R_{p t}\right\} P\left\{R_{4}^{\prime}>R_{s t}\right\} P\left\{\frac{1}{2} R_{1}>R_{p t}\right\} \\
+P\left\{R_{2}^{\prime}<R_{p t}\right\} P\left\{R_{4}^{\prime}<R_{s t}\right\} P\left\{\frac{1}{2} R_{1}>R_{p t}\right\} \\
+P\left\{R_{2}^{\prime}>R_{p t}\right\} P\left\{R_{4}^{\prime}>R_{s t}\right\} P\left\{R_{5}^{\prime}>R_{s t}\right\} \\
P\left\{R_{11}^{\mathrm{MRC}}>R_{p t}\right\}+P\left\{R_{2}^{\prime}>R_{p t}\right\} P\left\{R_{4}^{\prime}>R_{s t}\right\} \\
\times P\left\{R_{5}^{\prime}<R_{s t}\right\} P\left\{R_{12}^{\mathrm{MRC}}>R_{p t}\right\}
\end{array}\right\}
$$

For the secondary system, the outage probability is given by:

$$
P_{\text {out }}^{s^{\prime}}=1-P\left(R_{2}^{\prime}>R_{p t}\right) P\left(R_{3}^{\prime}>R_{p t}\right) P\left(R_{4}^{\prime}>R_{s t}\right) P\left(R_{7}^{\prime}>R_{s t}\right)
$$

where

$$
\begin{aligned}
R_{f}^{\prime} & =\frac{1}{2} \log _{2}\left(1+\frac{p_{p} r_{f}}{\sigma^{2}}\right)(f=2,3), \\
R_{g}^{\prime} & =\frac{1}{2} \log _{2}\left(1+\frac{p_{s} r_{g}}{\sigma^{2}}\right)(g=4,5), \\
R_{7}^{\prime} & =\frac{1}{2} \log _{2}\left(1+\frac{(1-\alpha) p_{r} r_{7}}{\sigma^{2}}\right), \\
R_{11}^{\mathrm{MRC}^{\prime}} & =\frac{1}{2} \log _{2}\left(1+\frac{p_{p} r_{1}}{\sigma^{2}}+\frac{\alpha p_{r} r_{6}}{\sigma^{2}}\right), \\
R_{12}^{\mathrm{MRC}} & =\frac{1}{2} \log _{2}\left(1+\frac{p_{p} r_{1}}{\sigma^{2}}+\frac{\alpha p_{r} r_{6}}{(1-\alpha) p_{r} r_{6}+\sigma^{2}}\right) .
\end{aligned}
$$

Here, the value $1 / 2$ is due to the fact that the whole transmission process is divided into two transmission phases.

\section{Simulation results and discussions}

The MATLAB tool is used to simulate the outage probabilities of the primary system and the secondary system under various system parameters. The topology of the cognitive relay network is constructed like this: the PT, PR, ST, SR, and Relay are collinear. In the twodimensional plane, the PT and the PR are located at the points $(0,0)$ and $(1,0)$, respectively. The Relay moves on the positive $\mathrm{X}$-axis. The $\mathrm{ST}$ is located at the midpoint of the PT and the Relay, while the SR is located at the midpoint of the Relay and the PR. In this topology, the parameters are set as $R_{p t}=R_{s t}=1, v=4$, $v 1=0.2, p_{p}=p_{r}=p_{s}=10, \sigma^{2}=1, d_{1}=1, d_{3}=0.5\left(1+d_{2}\right)$, $d_{4}=0.5 d_{2}, d_{5}=1-0.5 d_{2}, d_{6}=\left|1-d_{2}\right|, d_{7}=0.5\left|1-d_{2}\right|$, $d_{8}=0.1, \beta=0.5$, Ith $=3$. The values of $d_{2}$ are chosen as 0.5 and 1.2 , respectively. $\beta$ is the interference cancellation factor at the Relay. It is easy to learn that smaller $\beta$ leads to better outage performance. Without loss of 
generality, $\beta$ is chosen as 0.5 . The theoretical and numerical results for outage probabilities of the primary system and the secondary system versus the power allocation factor $\alpha$ are shown in Figure 2. Obviously, the theoretical results match well with the numerical results. For the cases of both $d_{2}=0.5$ and $d_{2}=1.2$, outage probabilities of the primary system and the secondary system in scheme A are lower than the outage probabilities of the systems in scheme B. It means that the outage performance of the primary system and the secondary system benefits from the full-duplex relay. In addition, for the case of $d_{2}=1.2$, the outage probabilities of the systems are higher than the ones for the case of $d_{2}=0.5$. Through analysis, we know that the Relay is able to assist the transmissions of the primary system and the secondary system. But for the case of $d_{2}=1.2$, the distance between the PT and the Relay is farther than that between the PT and the PR, and the benefit of cooperation from the Relay is not obvious compared with the introduced interference.

There exist two extreme cases, i.e., $\alpha=0$ and $\alpha=1$. The former corresponds to the protocol where the primary signal is only transmitted from the PT to the PR, and the transmission power of the Relay is used to transmit the secondary signal, which is regarded as an interfering signal at the PR. It is easy to get that outage performance of the secondary system is well, but outage probability of the primary system is unsatisfactory. With the increase of $\alpha$, outage performance of the primary system becomes better, and it has no obvious effect on outage performance of the secondary system. The latter refers to the protocol that the relay only helps the transmission of the primary system. So outage probability of the secondary system approaches 1 , which means that the ST cannot communicate with the SR. In this way, we are able to balance outage performances of the primary system and the secondary system though the power allocation factor $\alpha$.

It is easy to learn that outage probabilities of the primary system and the secondary system are affected by the variation of the interference cancellation factor $\beta$. So we show the effect of $\beta$ on outage performances of the primary system and the secondary system in Figure 3. For the case of $\beta=0$, it is said that self-interference is completely eliminated, and the outage probability of the primary system and the secondary system is very low. However, it is impossible in practical environments. With the increase of $\beta$, outage probabilities of the primary system and the secondary system become higher. When $\beta=1$, there is no self-interference cancellation at the relay input. For the primary system, because the relay keeps silent and $x_{p}$ is transmitted only through the direct link, outage probability of the primary system is very high. For the secondary system, because there is no direct link between the ST and the SR, outage probability of the secondary system approaches 1 , which means that the ST cannot communicate with the SR.

Then, we simulate the effects of transmission powers on outage performances. Outage probabilities of the primary system and the secondary system versus the transmission powers $p_{n} p_{p}$, and $p_{s}$ are shown in Figures 4, 5, and 6 , respectively. Here, we adopt the same topological structure and the same system parameters as before. Meanwhile, when one of $p_{n} p_{p}$, and $p_{s}$ is regarded as a variable, the other two parameters are set to 10. From

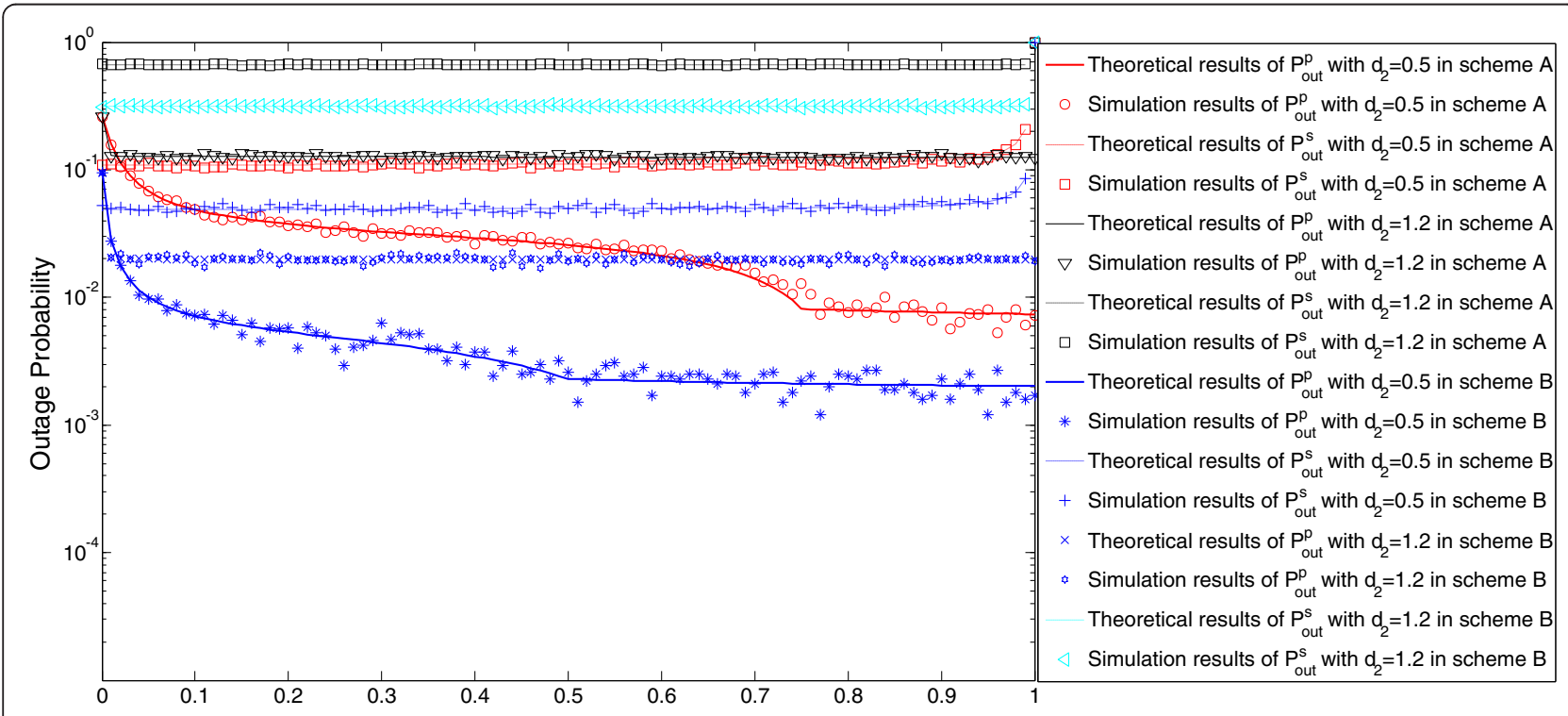

Figure 2 Outage probability versus power allocation factor $a$. 


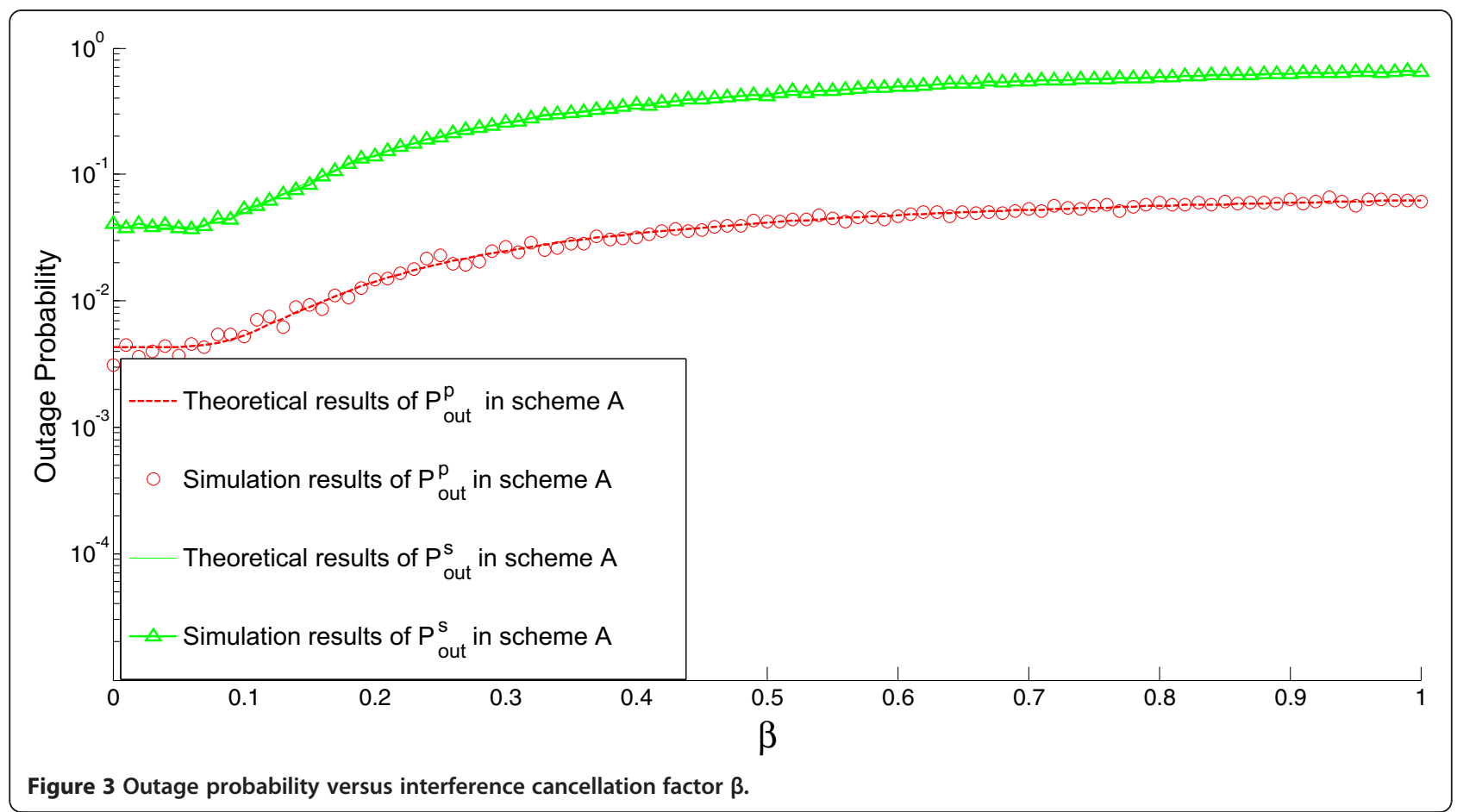

Figure 4 , with the increase of $p_{n}$ the cooperation capability and the mutual interference become more and more stronger, and the power of the self-interference, which affects the decoding at the Relay, becomes larger, too. So, outage probabilities of the primary system and the secondary system first become smaller and then become larger, and finally tend to be stable.
In Figure 5, we show the effect of transmission power of the primary transmitter $p_{p}$ on outage performances. Through analysis, we know that $P_{\text {out }}^{p}$ and $P_{\text {out }}^{s}$ decrease with the increase of $p_{p}$, and they match the simulation results in Figure 5. Because $p_{p}$ is the transmission power of the PT, outage probability of the primary system will decrease with the increase of $p_{p}$. For the secondary

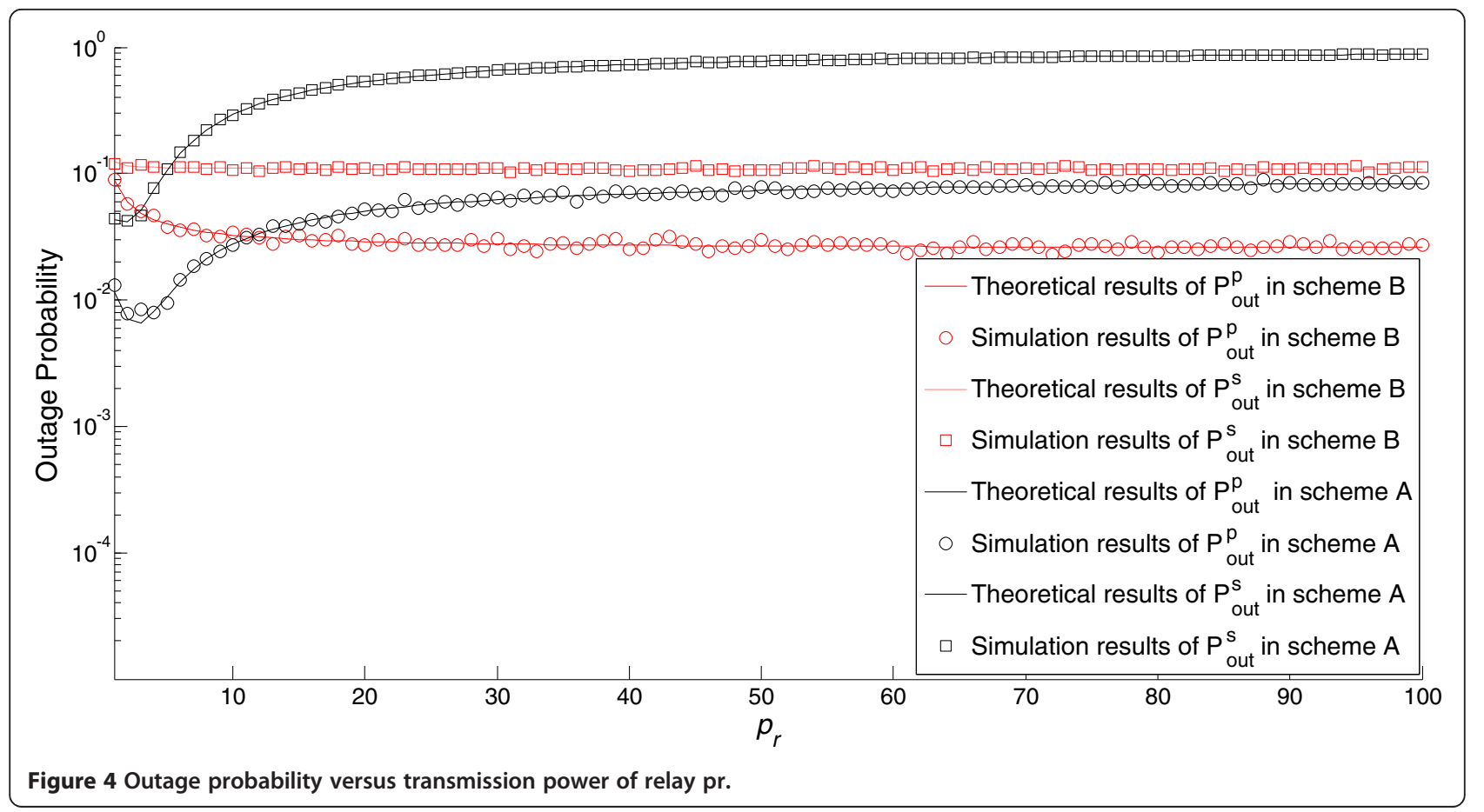




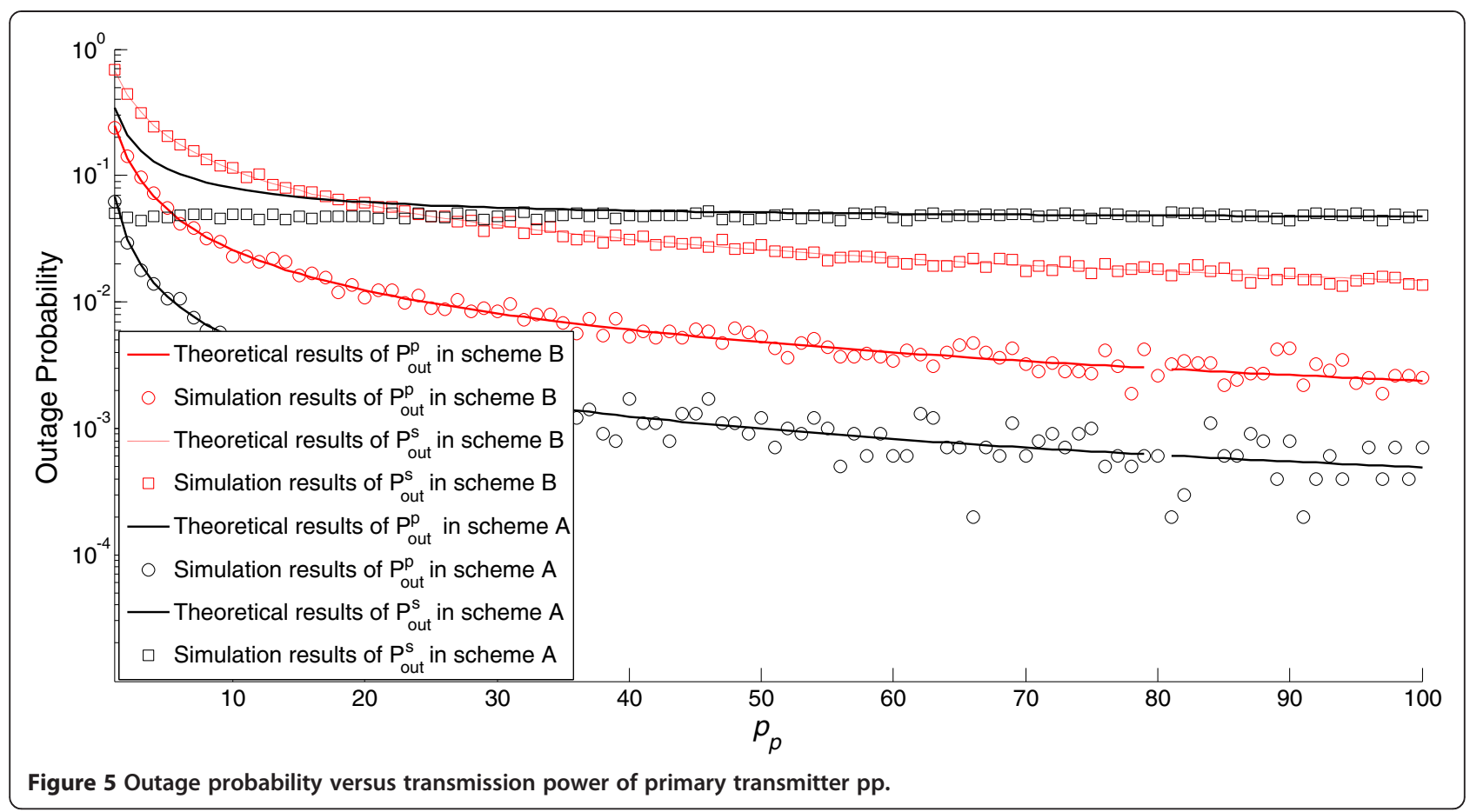

system, the decoding of the primary signal benefits from the increase of $p_{p}$, so outage probability of the secondary system also decreases with the increase of $p_{p}$.

The effect of transmission power of the secondary transmitter $p_{s}$ on outage performances are shown in Figure 6. It can be seen that both $P_{\text {out }}^{p}$ and $P_{\text {out }}^{s}$ decrease with the increase of $p_{s}$. For the secondary system, $P_{\text {out }}^{s}$ decreases with the increase of $P_{s}$, even though there is no obvious diminution of $P_{\text {out }}^{s}$. The reason is that there is no direct link between the ST and the SR. For the primary system, the interference caused by the secondary system will become larger, but the increase of $p_{s}$ also contributes to the decoding of secondary signal at the PR and the Relay. That is to say, the benefit of

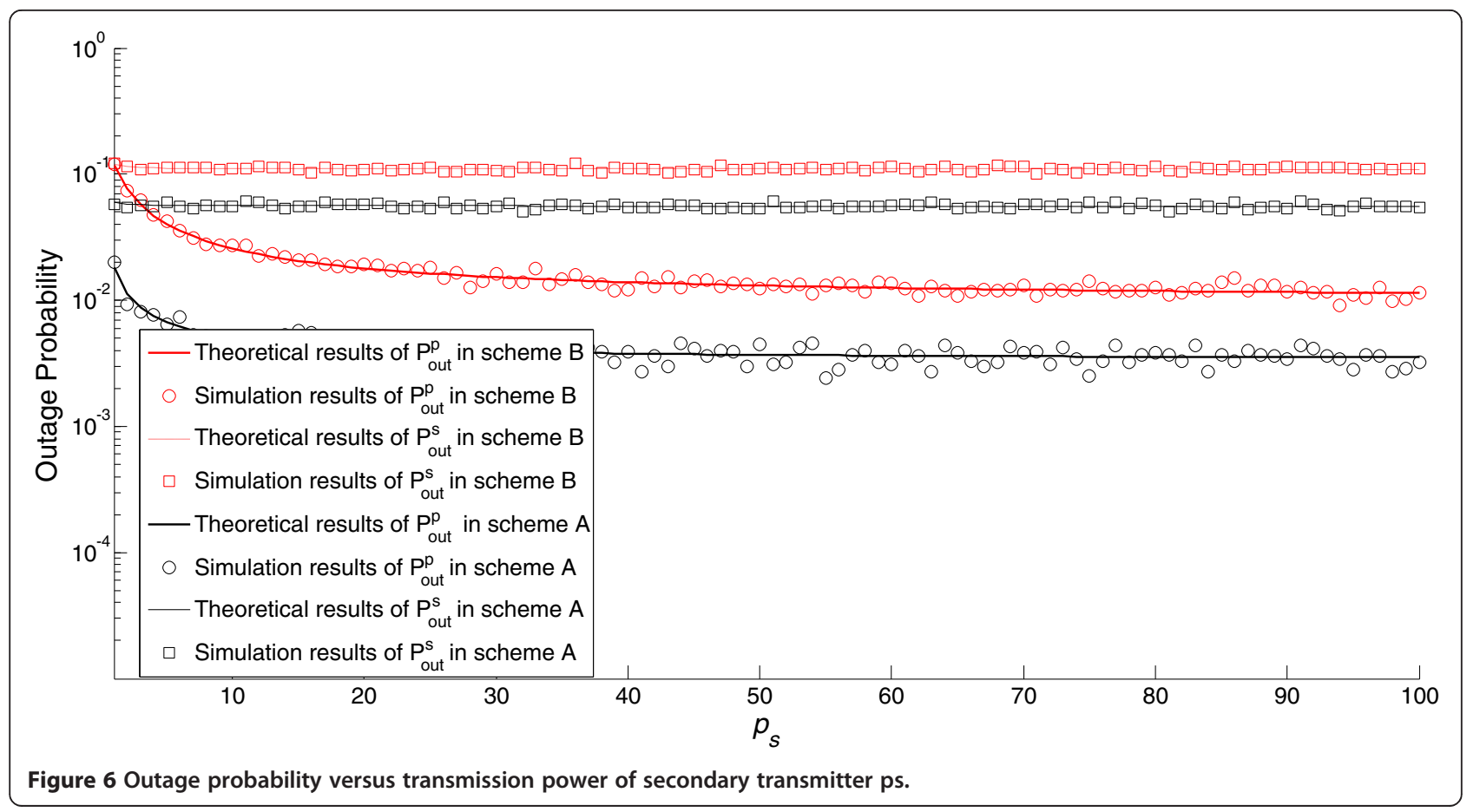


cooperation is far more than the interference when $p_{s}$ increases. So there is an obvious improvement on outage performance of the primary system with the increase of $p_{s}$

\section{Conclusions}

In this paper, outage performances of the primary system and the secondary system in a cognitive full-duplex relay network have been studied. The relay assists the transmissions of the primary system and the secondary system simultaneously. Self-interference was incorporated into the analysis which differentiate this work from outage performance analysis of cognitive half-duplex relay networks or full-duplex relay networks without spectrum sharing. Compared with the cognitive relay network adopting half-duplex relaying, better outage performances of the primary system and the secondary system are achieved under the assistance of the fullduplex decode-and-forward relay.

\section{Competing interests}

The authors declare that they have no competing interests.

\section{Acknowledgements}

This research was supported by the National Natural Science Foundation of China $(61162008,61172055)$, the Guangxi Natural Science Foundation (2013GXNSFGA019004), the Open Research Fund of Guangxi Key Lab of Wireless Wideband Communication and Signal Processing (12103), and the Director Fund of Key Laboratory of Cognitive Radio and Information Processing (Guilin University of Electronic Technology), Ministry of Education, China (2013ZRO2).

Received: 29 September 2014 Accepted: 7 January 2015

Published online: 01 February 2015

\section{References}

1. FCC Frequency Allocation Chart (National Telecommunications and Information Administration, 2003), http://www.ntia.doc.gov/osmhome/ allochrt.pdf (September 27, 2014)

2. Y Zou, B Zheng, J Zhu, Outage analysis of opportunistic cooperation over Rayleigh fading channels. IEEE Trans. Wireless Comm. 8, 3077-3085 (2009)

3. Y Zou, B Zheng, W Zhu, An opportunistic cooperation scheme and its BER analysis. IEEE Trans. Wireless Comm. 8, 4492-4497 (2009)

4. Y Zou, J Zhu, B Zheng, Y Yao, An adaptive cooperation diversity scheme with best-relay selection in cognitive radio networks. IEEE Trans. Signal Process. 58, 5438-5445 (2010)

5. R Mo, YH Chew, C Yuen, Information rate and relay precoder design for amplify-and-forward MIMO relay networks with imperfect channel state information. IEEE Trans. Veh. Tech. 61, 3958-3968 (2012)

6. V Asghari, S Aïssa, End-to-end performance of cooperative relaying in spectrum sharing systems with quality of service requirements. IEEE Trans. Veh. Tech. 60, 2656-2668 (2011)

7. S Haykin, Cognitive radio: brain-empowered wireless communications. IEEE J. Sel. Area. Comm. 23, 201-220 (2005)

8. Y Han, A Pandharipande, SH Ting, Cooperative decode-and-forward relaying for secondary spectrum access. IEEE Trans. Wireless Comm. 8, 4945-4950 (2009)

9. Y Han, SH Ting, A Pandharipande, Cooperative spectrum sharing protocol with secondary user selection. IEEE Trans. Wireless Comm. 9, 5438-5445 (2010)

10. H Ju, E Oh, D Hong, Improving efficiency of resource usage in two-hop full duplex relay systems based on resource sharing and interference cancellation. IEEE Trans. Wireless Comm. 8, 3933-3938 (2009)

11. T Kwon, S Kim, S Choi, D Hong, Optimal duplex mode for DF relay in terms of the outage probability. IEEE Trans. Veh. Tech. 59, 3628-3634 (2010)
12. JN Laneman, DNC Tse, GW Wornell, Cooperative diversity in wireless networks: efficient protocols and outage behavior. IEEE Trans. Inform. Theor 50, 3062-3080 (2004)

13. Z Ding, I Krikidis, B Rong, JS Thompson, C Wang, On combating the halfduplex constraint in modern cooperative networks: protocols and techniques. IEEE Wireless Comm. 19, 20-27 (2012)

14. C Zhong, HA Suraweera, A Huang, Z Zhang, C Yuen, Outage probability of dual-hop multiple antenna AF relaying systems with interference. IEEE Trans. Comm. 61, 108-119 (2013)

15. F Zhao, X Sun, H Chen, R Bie, Outage performance of relay-assisted primary and secondary transmissions in cognitive relay networks. EURASIP J. Wireless Commun. Netw. 2014, 60 (2014). doi:10.1186/1687-1499-2014-60

16. TM Cover, JA Thomas, Elements of Information Theory, 2nd edn. (John Wiley and Sons, New York, 2012)

17. T Riihonen, S Werner, R Wichman, Optimized gain control for singlefrequency relaying with loop interference. IEEE Trans. Wireless Commun. 8, 2801-2806 (2009)

18. T Cover, AE Gamal, Capacity theorems for the relay channel. IEEE Trans. Inform. Theor. 25, 572-584 (1979)

19. AE Gamal, M Mohseni, S Zahedi, Bounds on capacity and minimum energyper-bit for AWGN relay channels. IEEE Trans. Inform. Theor 52, 1545-1561 (2006)

20. M Duarte, C Dick, A Sabharwal, Experiment-driven characterization of fullduplex wireless systems. IEEE Trans. Wireless Comm. 11, 4296-4307 (2012)

21. T Riihonen, S Werner, R Wichman, Hybrid full-duplex/half-duplex relaying with transmit power adaptation. IEEE Trans. Wireless Comm. 10, 3074-3085 (2011)

22. T Riihonen, S Werner, R Wichman, Mitigation of loopback self-interference in full-duplex MIMO relays. IEEE Trans. Signal Process. 59, 5983-5993 (2011)

23. I Krikidis, H Suraweera, P Smith, C Yuen, Full-duplex relay selection for amplify-and-forward cooperative networks. IEEE Trans. Wireless Comm. 11, 4381-4393 (2012)

24. T Baranwal, D Michalopoulos, R Schober, Outage analysis of multihop fullduplex relaying. IEEE Comm. Lett. 17, 63-66 (2013)

25. I Krikidis, H Suraweera, S Yang, K Berberidis, Full-duplex relaying over block fading channel: a diversity perspective. IEEE Trans. Wireless Comm. 11, 4524-4535 (2012)

26. LJ Rodriguez, NH Tran, T Le-Ngoc, Optimal power allocation and capacity of full-duplex AF relaying under residual self-interference. IEEE Wireless Comm. Lett 3, 233-236 (2014)

27. LJ Rodriguez, NH Tran, T Le-Ngoc, Performance of full duplex AF relaying in the presence of residual self-interference. IEEE J. Sel. Area. Comm. 32, 1752-1764 (2014). Doi: 10.1109/JSAC.2014.2330151

28. G Zheng, I Krikidis, B Ottersten, Full-duplex cooperative cognitive radio with transmit imperfections. IEEE Trans. Wireless Commun. 12, 2498-2511 (2013)

29. HA Suraweera, I Krikidis, G Zheng, C Yuen, PJ Smith, Low-complexity endto-end performance optimization in MIMO full-duplex relay systems. IEEE Trans. Wireless Comm 13, 913-927 (2014)

30. H Kim, S Lim, H Wang, D Hong, Optimal power allocation and outage analysis for cognitive full duplex relay systems. IEEE Trans. Wireless Comm. $11,3754-3765$ (2012)

\section{Submit your manuscript to a SpringerOpen ${ }^{\odot}$ journal and benefit from:}

- Convenient online submission

Rigorous peer review

- Immediate publication on acceptance

- Open access: articles freely available online

- High visibility within the field

- Retaining the copyright to your article

Submit your next manuscript at $\gg$ springeropen.com 